\title{
Investigation on Texture Changes and Classification between Cold-Fresh and Freeze-Thawed Tan Mutton
}

\author{
Dongdong Li $\mathbb{D},{ }^{1}$ Yaling Peng $\mathbb{D}{ }^{1},{ }^{1}$ and Haihong Zhang $\mathbb{C}^{1,2}$ \\ ${ }^{1}$ School of Agriculture, Ningxia University, Yinchuan 750021, China \\ ${ }^{2}$ Ningxia Key Laboratory for Food Microbial Applications Technology and Safety Control, Ningxia University, \\ Yinchuan 750021, China \\ Correspondence should be addressed to Haihong Zhang; nxdwjyxx@126.com
}

Received 10 January 2019; Accepted 20 March 2019; Published 28 April 2019

Guest Editor: Gonzalo Delgado-Pando

Copyright (c) 2019 Dongdong Li et al. This is an open access article distributed under the Creative Commons Attribution License, which permits unrestricted use, distribution, and reproduction in any medium, provided the original work is properly cited.

To study the texture, microstructural changes, and classification of cold-fresh (C-F), freeze-thawed once (F- $\left.\mathrm{T}_{0}\right)$, and freeze-thawed twice Tan mutton $\left(\mathrm{F}-\mathrm{T}_{\mathrm{t}}\right)$, the aforementioned three types of Tan mutton were subjected to near-infrared hyperspectrum scanning, scanning electron microscopy, and TPA testing. The original spectrum of Tan mutton was obtained at a wavelength range of 900 1,700 nm after hyperspectrum scanning; a spectrum fragment ranging from $918 \mathrm{~nm}$ to 1,008 nm was intercepted, and the remaining original spectrum was used as a studied spectrum ("full spectrum" hereafter). The full spectrum was pretreated by SNV (standard normal variate), MSC (multiple scattering correction), and SNV + MSC and then extracted feature wavelengths by SPA (successive projections algorithm) and CARS (competitive adaptive reweighted sampling) algorithm, and 25 feature wavelengths were obtained. By combining these feature wavelengths with classified variables, the SNV + MSC-CARS-PLS-DA (partial least squares-discriminate analysis, PLS-DA) and SNV + MSC-SPA-PLS-DA models for classification of C-F and F-T Tan mutton were established. In contrast, SNV + MSC-CARS-PLS-DA yielded the highest classification rate of $98 \%$ and $100 \%$ for calibration set and validation set, respectively. The results indicated that the texture and surface microstructure of F-T Tan mutton deteriorated, and more worsely with F-T time. SNV+MSC-CARS-PLS-DA could be well used to classify C-F, F-T0, and F-Tt Tan mutton.

\section{Introduction}

Tan sheep (Yanchi, Ningxia, China) is a representative meat sheep breed with Chinese regional characteristics, even fat distribution, minimal mutton taste, tender meat, and reasonable nutritional composition [1]. However, due to deep processing and low level of conversion technology, more than $90 \%$ of Tan mutton is sold as cold-fresh $(\mathrm{C}-\mathrm{F})$ and frozen Tan mutton [2].

Freezing is the most effective means of preservation in the circulation and sale of Tan mutton products [3]. In addition to effectively inhibiting microbial growth and prolonging shelf life, it can easily lead to some unexpected changes in Tan mutton products such as the destruction of microstructure, loss of cell sap, and deterioration of texture. Moreover, the taste, flavor, and nutrient levels decrease after freeze-thaw (FT) treatment [4]. Criminals use F-T Tan mutton instead of C-F Tan mutton in selling for illegal profit [5].
Therefore, it is critical to develop a feasible and reliable technique that can be used for discrimination between C-F and F-T Tan mutton. In general, it is difficult to differentiate between C-F and F-T Tan mutton due to their similar appearance in shape, color, and texture [6]. To date, correlational analyses have been conducted to investigate the differences between C-F and F-T Tan meat using different approaches, including in enzymatic [7, 8], physiological [9], physical $[6,10]$, chemical $[6,10,11,12]$, and microbiological $[11,13]$ methods.

NIRS is a well-consolidated analytical technology that can fuse spectral information with component and surface sample information, thus precisely reflecting the "inside and outside" comprehensive changes [4]. Several hyperspectral techniques have been applied to the field of component analysis and F-T meat classification [14-16].

The hyperspectral imaging technique was investigated for nondestructive determination of refrigeration time and 
moisture content in chilled Tan mutton within the wavelength range of 400-1,000 $\mathrm{nm}$ [17]. Jin [18] proposed that the BP-ANN (back propagation-artificial neural network) quantitative prediction model for fat and protein content of fresh Tan mutton using the hyperspectral technique (wavelength: 900-1,700 nm), which showed that the PSOBP-ANN (established based on feature wavelengths extracted by PSO; particle swarm optimization, PSO) allows better prediction for fat and protein content in fresh Tan mutton. Furthermore, PLSR and LDA (linear discriminant analysis) classification models based on nine spectrum input variables (nine feature wavelengths extracted using the PLSR weight method) were applied to roughly discriminate tenderness grades of fresh Tan lamb [19] and achieve the desired discriminant results.

However, despite these investigations, no relevant study on the texture, microstructural changes, and classification of F-T Tan mutton has been conducted, to date. The specific aims of this study were (1) to perform scanning electron microscope (SEM) scanning to analyze the surface micro-

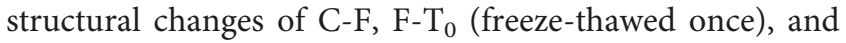
$\mathrm{F}_{-} \mathrm{T}_{\mathrm{t}}$ (freeze-thawed twice) Tan mutton; (2) to measure the hardness, gumminess, cohesiveness, springiness, chewiness, and resilience of above three types of Tan mutton to analyze their internal textural changes; (3) to collect spectral information and extract feature wavelengths of C-F, F- $\mathrm{T}_{0}$, and $\mathrm{F}_{-} \mathrm{T}_{\mathrm{t}}$ Tan mutton; and (4) to establish and select classified models based on feature wavelengths.

\section{Materials and Methods}

2.1. Sample Preparation. Samples were derived from sixmonth-old Yanchi (Ningxia, China) Tan mutton. The Tan sheep were slaughtered, and their hindquarters were eviscerated and packed with clean, transparent polyethylene film, and then transported $\left(-4^{\circ} \mathrm{C}\right.$, constant temperature $)$ to the laboratory within six hours.

Each eviscerated hindquarter was cut along the vertical muscle fiber direction into 213 pieces of $5 \mathrm{~cm} \times 5 \mathrm{~cm} \times 1 \mathrm{~cm}$ (length $\times$ width $\times$ height) and used as testing samples at room temperature $\left(25^{\circ} \mathrm{C}\right)$. The average weight of the sample was approximately $49.50 \mathrm{~g}$, and the weight ranges from $40.68 \mathrm{~g}$ to $59.21 \mathrm{~g}$ (AB104-N, Mettler Toledo, Shanghai, China).

The above 213 samples were randomly divided into the C-F Tan mutton group (rapid cooling treatment, with center temperature of the sample dropping to $0-4^{\circ} \mathrm{C}$ within $24 \mathrm{~h}$ ), $\mathrm{F}_{-} \mathrm{T}_{\mathrm{o}}$ group (rapid cooling treatment; samples were frozen at $-23^{\circ} \mathrm{C}$ for $36 \mathrm{~h}$ and thawed at $25^{\circ} \mathrm{C}$ for $2 \mathrm{~h}$ ), and $\mathrm{F}-\mathrm{T}_{\mathrm{t}}$ group (rapid cooling treatment; $\mathrm{F}_{-} \mathrm{T}_{\mathrm{o}}$ reprocessed twice, samples were frozen at $-23^{\circ} \mathrm{C}$ for $36 \mathrm{~h}$ and thawed at $25^{\circ} \mathrm{C}$ for $2 \mathrm{~h}$, and then frozen at $-23^{\circ} \mathrm{C}$ for $36 \mathrm{~h}$ and thawed at $25^{\circ} \mathrm{C}$ for $2 \mathrm{~h}$ ). A total of 210 samples were used for spectral image acquisition and texture measurement, and the remaining three samples were used for scanning electron microscopy.

To avoid the influence of thawing moisture on the absorption efficiency of the sample, filter paper was used to absorb the surface moisture of the sample before the spectral imaging.
2.2. Spectral Image Acquisition. The hyperspectral images of the Tan mutton were acquired using a hyperspectral collecting system (wavelength range: 900-1,700 nm, spectral resolution: $5 \mathrm{~nm}, 256$ bands). The system consisted of a CCD camera (1,004 pixels $\times 668$ pixels, Zelos-285 GV, Kappa Optronics GmbH, Gleichen, Germany), Inspector (N17 E Spectral Imaging, Ltd., Oulu. Finland), slit width: $30 \mu \mathrm{m}$, slit length: $9.6 \mathrm{~mm}, 35 \mathrm{~W}$ tungsten lamp light source (HSIA-LSTDIF, Zolix Instruments Co., Ltd., Beijing, China), electronic control displacement platform (PSA200-11-X), and computer (Lenovo Intel ${ }^{\circledR}$ Core i7-2600CPU@3. $4 \mathrm{GHz}$, RAM4. 00G) equipped with data acquisition software (SpectrumSENS, Zolix Instruments).

The hyperspectral collecting system was precalibrated before capturing spectral images of the samples. The steps were as follows: all-black images were obtained by covering the camera lens to eliminate the effect of the dark current in the camera. All-white images were obtained by collecting standard whiteboard images to eliminate the influence of uneven distribution of indoor light [20].

Tan mutton samples were placed on a piece of black light-absorbing cloth, the reflection of which was nearly zero at every wavelength, thereby decreasing the influence of reflection of the background during the process of hyperspectral image acquisition. To collect a nondistortional hyperspectral image, the speed of the stepper motor was adjusted to synchronize with the scanning speed. Hence, each sample moved at a constant speed of $10 \mathrm{~mm} / \mathrm{s}$ over the translation stage. The actual length of the scan line was $80 \mathrm{~mm}$ (clear sample images are available). The distance between the surface of the Tan mutton being imaged and the lens and the lamps were fixed at $385 \mathrm{~mm}$ and $300 \mathrm{~mm}$, respectively. The exposure time of the CCD camera was set to $10 \mathrm{~ms}$ (allows the best definition of the image). Hyperspectral images were acquired within the spectral range of $900 \sim 1,700 \mathrm{~nm}$, with $2.97 \mathrm{~nm}$ intervals between contiguous bands, with a total of 256 bands. The images were saved in a band-interleaved-by-line (BIL) format.

2.3. Measurement of Textural Indices. To better analyze the internal and external changes of the F-T Tan mutton, mechanical parameters, including hardness, gumminess, cohesiveness, springiness, chewiness, and resilience, were measured after acquiring hyperspectral images.

Fifteen samples were randomly selected from each of the three groups that were placed on the platform of the texture analyzer. The upper surface was a test contact plane, whose direction is parallel to the muscle fiber. Textural indices were measured by compressing four points on a cross equilateral line and one point in the middle position of the upper surface. The mean of five measurements was the final result.

Texture apparatus (TA.XT Plus, SMS Corp., England) equipped with a $\mathrm{P} / 36 \mathrm{R}$ probe was implemented by means of a TPA mode. The other parameters were set as follows: falling speed: $1 \mathrm{~mm} \cdot \mathrm{s}^{-1}$ before trigger; loading rate: $1 \mathrm{~mm} \cdot \mathrm{s}^{-1}$; rising speed: $5 \mathrm{~mm} \cdot \mathrm{s}^{-1}$ after the test; trigger force: $5 \mathrm{~g}$; data acquisition rate: 200 pps; deformation: $30 \%$; and measurement interval time: $5 \mathrm{~s}$. Hardness, gumminess, cohesiveness, 
springiness, chewiness, and resilience were obtained by analyzing the strength-time curve.

2.4. Scanning Electron Microscopy. The formation and growth of ice crystals in the muscle cell has a destructive effect on the tissue structure, including the cell membrane [21]. Therefore, it is necessary to analyze the microcosmic structural differences of the F-T Tan mutton.

The three samples that are selected from 213 samples were cut into $3 \mathrm{~mm} \times 1 \mathrm{~mm} \times 1 \mathrm{~mm}$ (length $\times$ width $\times$ height) sample strips along the direction of muscle fiber, then the sample strips were immediately observed to obtain highdefinition images via a field emission scanning electron microscope (15 kV:1 nm: Jsm-7500f03040701, Japan Electronic Co., Ltd.).

The experimental method was based on Haga and Ohashi [22] with minor modification: $0.1 \mathrm{~mol} / \mathrm{L}$ phosphate buffer ( $\mathrm{pH} \mathrm{7)}$ was used to prepare the glutaraldehyde solution and formaldehyde solution, with a volume fraction of $2.5 \%$ and $4 \%$, respectively. The two solutions were mixed at a $1: 1$ ratio and used in fixing the samples for $24 \mathrm{~h}$ at $4^{\circ} \mathrm{C}$. After fixation, the samples were rinsed with $0.1 \mathrm{~mol} / \mathrm{L}$ phosphate buffer ( $\mathrm{pH} 7$ ) for thrive, for $30 \mathrm{~min}$ each wash. The samples were then fixed in $1 \%$ osmium tetroxide solution $(0.1 \mathrm{~mol} / \mathrm{L}$ phosphate buffer, $\mathrm{pH} 7$ ) at $4^{\circ} \mathrm{C}$ for $2 \mathrm{~h}$, then rinsed with distilled water thrice, and then dehydrated across an ethanol gradient $(30 \%, 70 \%$, and $100 \%)$. The samples were observed and pasted on the SEM sample table after freeze-drying (ES2030 HITACHI) and sprayed using an ion sputtering film apparatus (E-1010 Giko). The samples were observed and photographed under a SEM.

2.5. Spectral Image Preprocessing. A total of 210 hyperspectral cube images (including two-dimensional spatial and spectral information) were collected based on the parameters described in Section 2.2.

To eliminate the uneven intensity distribution of light sources in each band and the nonspectral information introduced by sensor responses, raw images $\left(R_{0}\right)$ were corrected and images in reflectance mode $\left(R_{\mathrm{c}}\right)$ were obtained using the following formula:

$$
R(\%)=\frac{R_{0}-D}{W-D} \times 100,
$$

where $D$ is the dark reference image ( $\sim \%$ reflectance) obtained by covering the camera lens using the opaque cap and $\mathrm{W}$ is the white reference image ( $100 \%$ reflectance) obtained by a standard whiteboard. This step is called image calibration. Here, the dark and white reference images were regularly updated during the experiments.

After image calibration, the hyperspectral images were resized to reduce their irrelevant information. Based on the actual size of the sample, the pixels of the hyperspectral cubic image of each sample were adjusted from 1,004 pixels $\times 668$ pixels to 600 pixels $\times 480$ pixels in ENVI 4.8 (Research System, Inc., Boulder, CO, USA) software, then the whole sample surface ( 600 pixels $\times 480$ pixels) was selected as the region of interest (ROI), and the average reflection spectrum of ROI was extracted.

The average spectrum was pretreated using SNV, MSC, and $\mathrm{SNV}+\mathrm{MSC}$ methods to eliminate irrelevant information and noise (e.g., stray light, sample background, and electrical noise) contained in the spectrum as much as possible.

2.6. Extraction of Feature Wavelengths. The pretreated spectrum still contains a lot of redundant information, and the difference in effective spectrum is small, and thus it takes time to establish a model and weakens the performance of the model due to the introduction of invalid information. The CARS and SPA algorithms have their own advantages in selecting feature wavelengths and reducing data collinearity.

The CARS algorithm combines exponentially decreasing function (EDP) with adaptive reweighted sampling (ARS) to select the PLS subset model with the minimum RMSECV ( $80 \%$ of the classified variables that were randomly selected during each selection process to establish the PLS model calibration set model) [23]. The selected PLS subset model consists of optimal classified variables. Thus, the CARS algorithm has a significant advantage in selecting the most effective wavelengths.

The SPA can calculate the projection of the remaining wavelengths of the preselected variable combination and select out the smallest collinearity combination, thereby eliminating multicollinear wavelengths to the maximum extent [24].

2.7. Establishment of the PLS-DA Model. PLS-DA based on partial least squares (PLS) algorithm is powerful in solving nonlinear classification problems, noise reduction, and variable selection [25].

In this study, PLS-DA was applied on a dataset that consists of $X$ and $Y$ matrices. The $X$ matrix consisted of the spectral information [26], with $n$ being the gross of the spectrum and $p$ the quantity of classified variables. The $Y$ matrix contains $n$ rows and three columns that map three classes of Tan mutton, namely, C-F, F-T $\mathrm{T}_{\mathrm{o}}$, and $\mathrm{F}-\mathrm{T}_{\mathrm{t}}$ of the dataset.

The sample set partitioning based on the joint $X-Y$ distance (SPXY) method was used to partition the sample set before establishing PLS-DA, which can calculate each sample distance based on spectral and classified variables individually. The calculated sample distance was divided by its own maximum value so that it equals the weight of the two feature parameters when the sample is selected [27].

In this study, the sample ratio of calibration and validation set is $3: 1$. The distance between samples is defined by the following formula:

$$
\begin{aligned}
& d_{x y}(i, j)=\frac{d_{x}(i, j)}{\max _{i, j \in(1, z)\left[d_{x}(i, j)\right]}}+\frac{d_{y}(i, j)}{\left.\max _{i, j \in(1, z)\left[d_{y}(i, j)\right.}\right]}, \\
& i, j \in[1, z] \text {, }
\end{aligned}
$$


where $d_{x}(i, j)$ and $d_{y}(i, j)$ are the distance of each sample based on spectral and classified variables, respectively. A total of 160 samples were selected to set up the calibration model, and 50 samples were used to validate the prediction performance of the model.

\section{Results and Discussion}

3.1. Microstructural Analysis of C-F, F-T $T_{o}$, and F-T Tan Mutton. Figure 1 represents the SEM images of C-F, F- $\mathrm{T}_{\mathrm{o}}$, and $\mathrm{F}_{-} \mathrm{T}_{\mathrm{t}}$ Tan mutton. The muscle fiber surface of $\mathrm{C}-\mathrm{F}$ Tan mutton is smooth and clear, and the arrangement of muscle fiber is neat and regular. The compact muscle bundle gap of muscle fiber is smaller (Figure 1(a)). After F-T treatment (Figure 1(b)), the structure of the muscle fibers showed slight "deterioration", which includes slight contraction, deformity, slight protrusion on the surface, a decrease in smoothness of the muscle fiber, an increase in the gap among bundles, and variable degrees shape of muscle bundle distortion. These features may be attributable to the elasticity of the connective tissue membrane in muscles, which plays a role in maintaining muscle integrity and preventing muscle fiber damage [28]. During the freezing process, the formation and growth of ice crystals in the muscle cells caused crush damage to the connective tissue membrane. The internal structure integrity of the muscle cells was destroyed [29]. The smaller gaps in the muscle bundles were due to thawing and stiffening, and the sarcomere contracts $36 \mathrm{~h}$ after slaughter, thereby resulting in a decrease of muscle fiber gap and an increase of muscle bundle space. In addition, these changes may also be due to cold contraction of the carcass as these were immediately placed in a frozen environment (low temperature stimulates the neural conditioned reflex or the calcium pump in the sarcoplasmic reticulum is sensitive to low temperatures, which results in stronger interactions between actin and myosin) [30].

After $\mathrm{F}_{-} \mathrm{T}_{\mathrm{t}}$ treatment (Figure 1(c)), the muscle stiff mature tended to be completed and the muscles stiffen within $72 \mathrm{~h}$ of slaughter; the muscle fibers are severely deformed, broken, disorganized, and loosely structured, which have resulted from the destruction of myofibrillar skeleton and degradation of intermuscular line protein and troponin $\mathrm{T}[31]$.

Texture can directly reflect the mechanical properties and taste changes in $\mathrm{C}-\mathrm{F}, \mathrm{F}-\mathrm{T}_{\mathrm{o}}$, and $\mathrm{F}-\mathrm{T}_{\mathrm{t}}$ Tan mutton. Table 1 illustrates that the hardness $(253.12 \mathrm{~N}$ and $221.55 \mathrm{~N})$ and chewiness $\left(118.11 \mathrm{~N}\right.$ and $116.33 \mathrm{~N}$ ) of the $\mathrm{F}-\mathrm{T}_{\mathrm{o}}$ and $\mathrm{F}-\mathrm{T}_{\mathrm{t}}$ groups were significantly lower than those of the $\mathrm{C}-\mathrm{F}$ group $(323.70 \mathrm{~N}$ and $179.15 \mathrm{~N})(p<0.05)$. There was no significant difference $(p>0.05)$ in springiness between the $\mathrm{F}_{-} \mathrm{T}_{\mathrm{t}}$ and $\mathrm{C}-\mathrm{F}$ groups, but was significantly lower than that of the $\mathrm{F}-\mathrm{T}_{\mathrm{o}}$ group $(p<0.05)$. No significant difference $(p<0.05)$ in cohesiveness among the three groups was observed.

Hardness of Tan mutton relates to the content and distribution of protein and muscle fiber, which decreases with loss of muscle moisture [19]. After F-T treatment, cell structure was destroyed, cell sap was los,t and myofibrillar protein degraded, which resulted in a significant decrease in hardness.
The springiness of Tan mutton increases with moisture content to a certain extent [19]. It does not induce detectable damage to cell structure (compared with Figures 2(a) and 2(b)) and leads to slight exudation of muscle fiber cell sap after $\mathrm{F}_{-} \mathrm{T}_{\mathrm{o}}$ treatment, which may be the reason why $\mathrm{F}_{-} \mathrm{T}_{\mathrm{o}} \mathrm{Tan}$ mutton's springiness was higher than that of C-F Tan mutton.

The chewiness of Tan mutton is a comprehensive reflection of hardness, cohesiveness, and springiness [32]. Theoretically, springiness and hardness also affect the chewiness of Tan mutton, so the chewiness of C-F Tan mutton was significantly higher than that of the other two groups.

3.2. Spectral Pretreatment. Spectral absorbability is closely related to sample composition [33]. Figure 3 plots the original near-infrared spectral curve of the 210 samples. Due to minimal differences in the main components of C-F, F- $\mathrm{T}_{\mathrm{o}}$, and $\mathrm{F}-\mathrm{T}_{\mathrm{t}}$ Tan mutton, the trend of the spectral curve of each sample was similar and no distinct abnormal samples were observed.

The spectral curve within the wavelength range of 918 1,008 $\mathrm{nm}$ was chaotic because of the low signal-noise ratio of the hyperspectral camera, and it was easy to amplify the noise after calibration [34]. Thus, the spectral fragment ranging from $918 \mathrm{~nm}$ to $1,008 \mathrm{~nm}$ was intercepted, and the original spectrum containing the 225 bands and ranging from $1,010 \mathrm{~nm}$ to $1,678 \mathrm{~nm}$ was used for further investigation.

There were less different chemical components with samples from each group, and each chemical component showed relatively stable spectral absorption, which leads to the analogous ROI spectrum for each group of samples. Therefore, the average reflectance spectral curves of ROI ranging from 1,010 $\mathrm{nm}$ to 1,678 $\mathrm{nm}$ are shown in Figure 2(b), the increase and decrease trend is consistent, and the positions of their peaks and troughs are very close.

Figure 2(b) shows the following:

(1) The ROI spectral curves of C-F, F- $T_{o}$, and F- $T_{t}$ Tan mutton are similar, their trends of increase and decrease are consistent, and the locations of the peaks and troughs are very close. The general trend of the ROI spectral curves shows an oblique " $\mathrm{M}$ " shape within $1,065 \mathrm{~nm} \sim 1,415 \mathrm{~nm}$. Each curve has one peak at $1,150 \mathrm{~nm}$ and $1,325 \mathrm{~nm}$, a trough at $1,260 \mathrm{~nm}$, and a gentle curve trend within the spectral range of $1,440 \mathrm{~nm}$ to $1,650 \mathrm{~nm}$.

(2) The ROI average spectral reflectance of C-F Tan mutton at $1,010 \mathrm{~nm} \sim 1,415 \mathrm{~nm}$ was higher than that of F-T Tan mutton. A significant difference in ROI average spectral reflectance was observed among the three groups at 1,010 $\mathrm{nm} \sim 1,177 \mathrm{~nm}\left(\mathrm{C}-\mathrm{F}>\mathrm{F}-\mathrm{T}_{\mathrm{o}}>\mathrm{F}-\right.$ $\left.\mathrm{T}_{\mathrm{t}}\right)$.

(3) The ROI average spectral reflectance of C-F, F-T and $\mathrm{F}-\mathrm{T}_{\mathrm{t}}$ Tan mutton had the local minimum values at $1,065 \mathrm{~nm}$, which showed strong absorbability (protein denaturation and hydrolysis due to the 


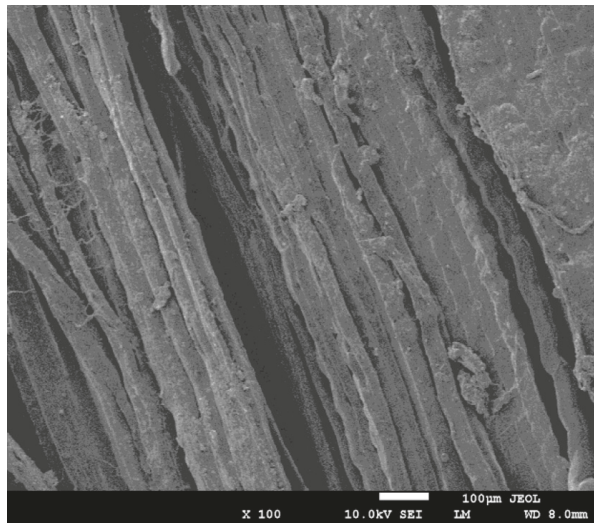

(a)

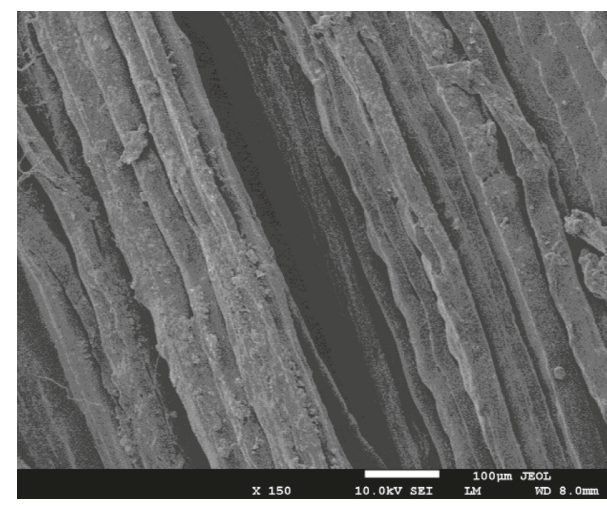

(b)

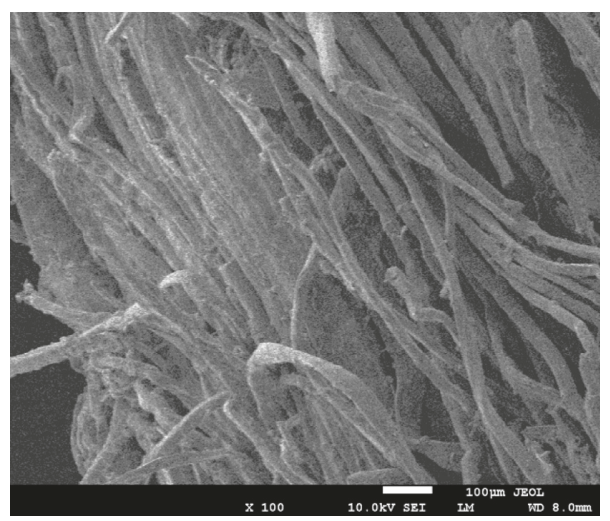

(c)

Figure 1: Scanning electron microscopy of Tan mutton. (a) C-F Tan mutton. (b) F-T Tan mutton. (c) F-T Tan mutton.

TABle 1: Textures of C-F, F- $\mathrm{T}_{\mathrm{o}}$, and $\mathrm{F}-\mathrm{T}_{\mathrm{t}}$ Tan mutton.

\begin{tabular}{lcccccc}
\hline Category & Hardness & Springiness & Cohesiveness & Gumminess & Chewiness & Resilience \\
\hline C-F Tan mutton & $323.7008 \pm 39.3047 \mathrm{a}$ & $0.8749 \pm 0.0674 \mathrm{~b}$ & $0.6281 \pm 0.0676 \mathrm{a}$ & $203.8078 \pm 36.2140 \mathrm{a}$ & $179.1529 \pm 38.0677 \mathrm{a}$ & $0.2278 \pm 0.0554 \mathrm{a}$ \\
$\mathrm{F}-\mathrm{T}_{\mathrm{o}}$ Tan mutton & $253.1150 \pm 49.2373 \mathrm{~b}$ & $1.5866 \pm 0.3850 \mathrm{a}$ & $0.6236 \pm 0.0549 \mathrm{a}$ & $158.6631 \pm 38.4950 \mathrm{~b}$ & $118.1115 \pm 39.1555 \mathrm{~b}$ & $0.2401 \pm 0.0331 \mathrm{a}$ \\
$\mathrm{F}_{\mathrm{T}}$ Tan mutton & $221.5507 \pm 54.7750 \mathrm{~b}$ & $0.8081 \pm 0.0677 \mathrm{~b}$ & $0.6547 \pm 0.0464 \mathrm{a}$ & $144.4275 \pm 32.4243 \mathrm{~b}$ & $116.3317 \pm 26.1189 \mathrm{~b}$ & $0.2374 \pm 0.0284 \mathrm{a}$ \\
\hline
\end{tabular}

Mean of three replicates \pm standard deviation; means in the same column with different letters are significantly different $(p<0.05)$. Reversely no difference $(p>0.05)$.

destruction of cell structure by crystallization, an increase in nitrogen-containing exudates may be related to the third order frequency-doubling of $\mathrm{N}-\mathrm{H}$ bond) and the local maximum values at $1,150 \mathrm{~nm}$, which showed strong reflectivity (accounting for the water bands after F-T treatments, which was possibly due to the water loss after being thawed).

SNV, MSC, and SNV + MSC were applied to pretreat the original spectrum. Comparative analysis indicated that $\mathrm{SNV}+\mathrm{MSC}$ is the best method, owing to the better performance of established PLS model based on spectral pretreatment by SNV + MSC. This may be due to an amount of spectrum noise being removed and increasing with the differences among original spectral variables after SNV + MSC pretreatment, which improves the robustness and performance of the PLS model. Therefore, the spectrum after SNV + MSC pretreatment (Figure 2(c)) was applied for further investigation.
3.3. Extraction of Feature Wavelengths. Before running CARS, the optimal principal component number of the PLS model was first determined. The maximum principal component number was set to 40 , and the sampling number of Monte Carlo was 200. Three quarters of the total samples were used as the calibration set, and the RMSECV under different the principal component numbers were obtained (Figure 3). When the principal component number is 18 , the minimum RMSECV is 0.2674 , so the optimal principal component number was 18 .

Setting CARS running parameters: The Monte Carlo sampling number is 200 , the principal component number was 18 , and cross-validation group number was 10 . The CARS running results are shown in Figures 4(a)-4(c), which represent the changes in the number of sampled variables, the RMSECV of 10 -fold cross validation and regression coefficient with the increasing number of $\mathrm{MC}$ sampling runs. 

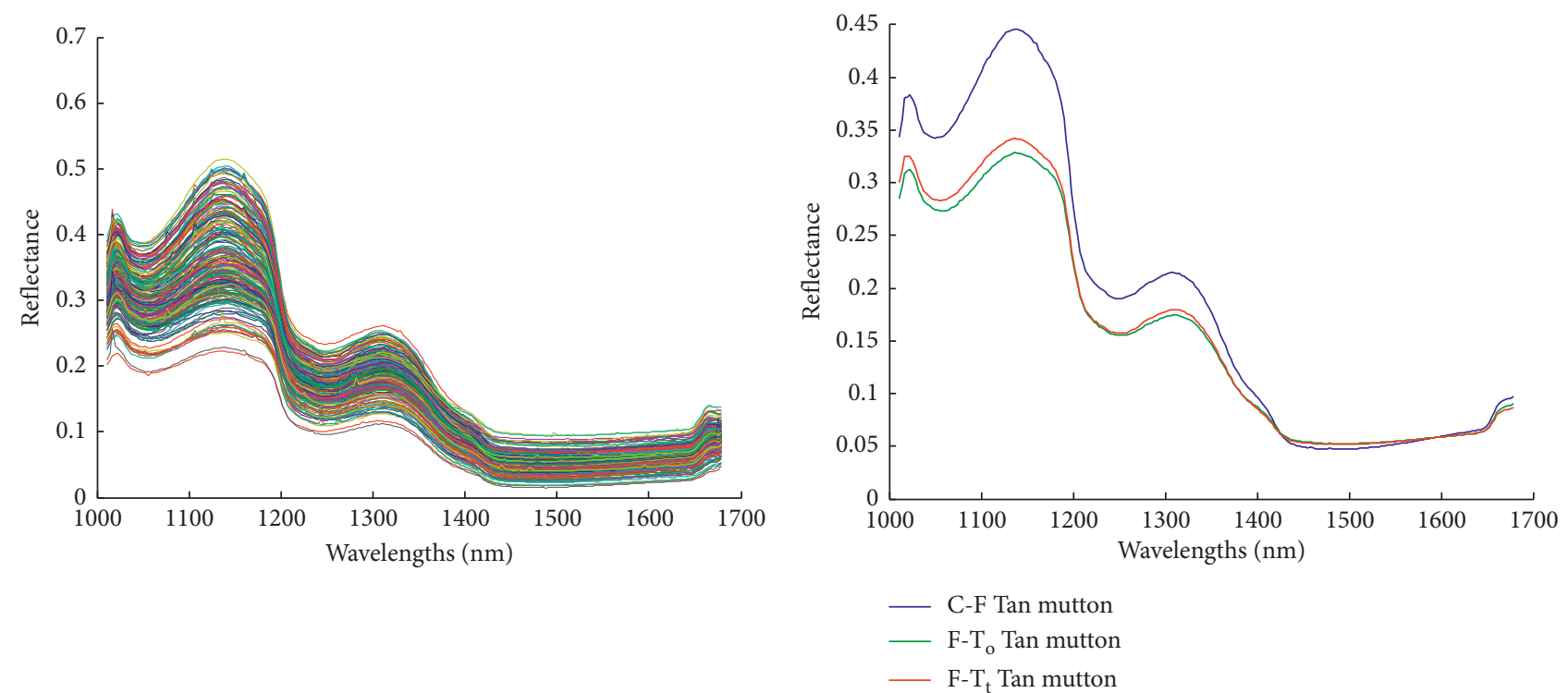

(a)

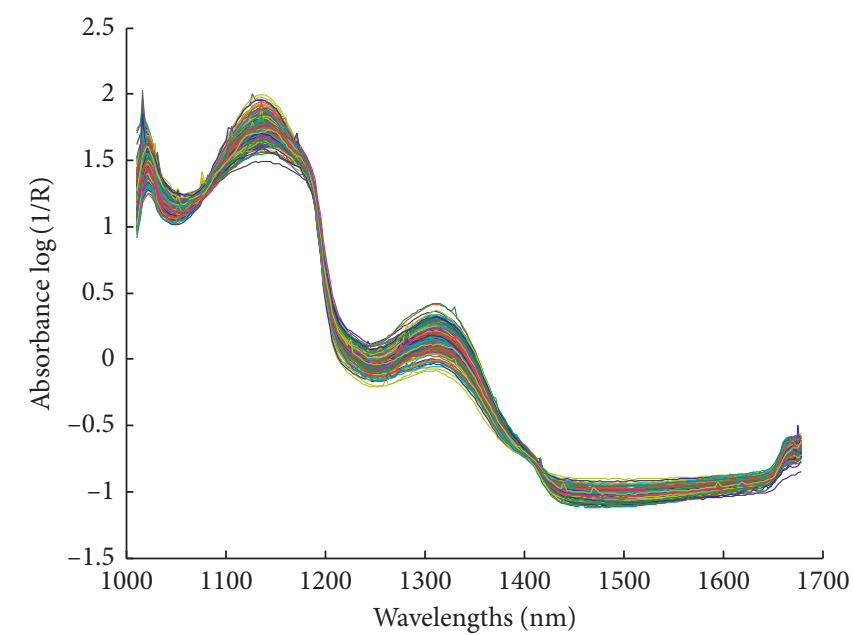

(c)

(b)

FIgURE 2: Spectral images. (a) Near-infrared original spectrum. (b) ROI average reflectance spectrum. (c) Spectrum preprocessed by SNV + MSC.

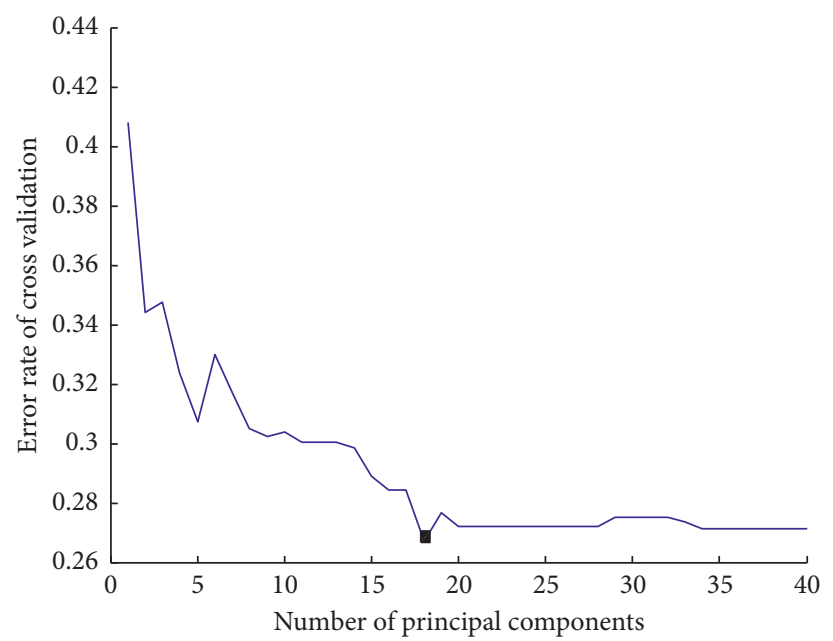

FIGURE 3: Variation in RMSECV values with principal numbers of the PLS model. 


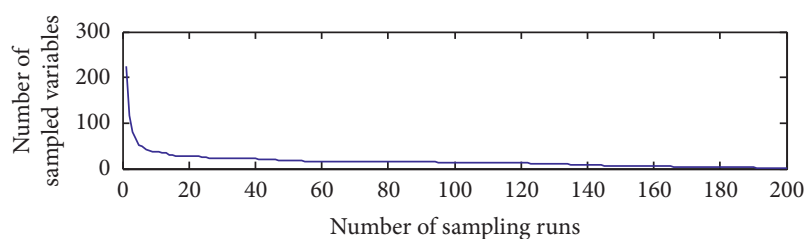

(a)

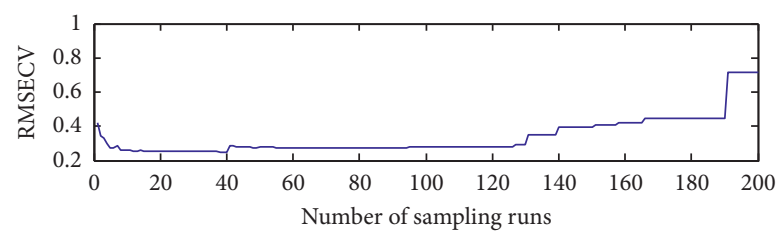

(b)

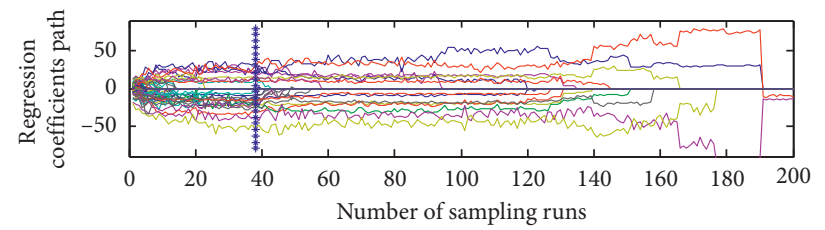

(c)

FIgURE 4: The result of feature wavelengths extracted by CARS.

Figure 4(a) shows that a rapid reduction in the number of sampled variables was observed in the first four MC sampling runs, and then gradually decreased, which shows the "rough" and "elaborate" selection process of key variables based on EDP [23].

Figure 4(b) shows that the RMSECV of the established PLS model that was based on selected variables becomes smaller with increasing MC sampling time in the first $38 \mathrm{MC}$ sampling runs, which indicates that a large amount of irrelevant and collinear information was eliminated. When the sampling run is 38 (located in the asterisk vertical line in Figure 4(c)), the RMSECV reaches a minimum value 0.2490 . After $38 \mathrm{MC}$ sampling runs, the RMSECV gradually increased with more MC sampling runs, which may be due to the removal of some important information from classified variables. Therefore, 23 feature wavelengths were obtained as follows: $1,052 \mathrm{~nm}$, $1,062 \mathrm{~nm}, 1,130 \mathrm{~nm}, 1,169 \mathrm{~nm}, 1,205 \mathrm{~nm}, 1,220 \mathrm{~nm}, 1,240 \mathrm{~nm}$, $1,300 \mathrm{~nm}, 1,324 \mathrm{~nm}, 1,327 \mathrm{~nm}, 1,357 \mathrm{~nm}, 1,366 \mathrm{~nm}, 1,372 \mathrm{~nm}$, $1,398 \mathrm{~nm}, 1,401 \mathrm{~nm}, 1,413 \mathrm{~nm}, 1,443 \mathrm{~nm}, 1,488 \mathrm{~nm}, 1,544 \mathrm{~nm}$, $1,571 \mathrm{~nm}, 1,628 \mathrm{~nm}, 1,643 \mathrm{~nm}$, and $1,655 \mathrm{~nm}$.

To identify the optimal method for screening feature variables, the SPA is also used as a comparison algorithm to extract the feature wavelengths from $1,010 \mathrm{~nm}$ to $1,678 \mathrm{~nm}$ so that it can minimize the collinearity among selected variables. The SPA running parameters were set as follows: the selected number of feature wavelengths ranged from 1 to 35 , and the step length was 1 . The results are shown in Figure 5. Figure 5(a) shows that RMSE varies with the number of variables included in the model, which indicates the dynamically eliminated process of collinear variables. When the RMSE was 0.2560 , the number of collinear variables included in model was lowest, and 23 feature wavelengths were selected from Figure 5(b): $1,011 \mathrm{~nm}, 1,017 \mathrm{~nm}, 1,032 \mathrm{~nm}, 1,053 \mathrm{~nm}$, $1,056 \mathrm{~nm}, 1,076 \mathrm{~nm}, 1,080 \mathrm{~nm}, 1,083 \mathrm{~nm}, 1,094 \mathrm{~nm}, 1,106 \mathrm{~nm}$, $1,136 \mathrm{~nm}, 1,160 \mathrm{~nm}, 1,181 \mathrm{~nm}, 1,190 \mathrm{~nm}, 1,226 \mathrm{~nm}, 1,243 \mathrm{~nm}$, $1,261 \mathrm{~nm}, 1,401 \mathrm{~nm}, 1,410 \mathrm{~nm}, 1,479 \mathrm{~nm}, 1,595 \mathrm{~nm}, 1,643 \mathrm{~nm}$, and $1,673 \mathrm{~nm}$.

3.4. Establishment of the PLS-DA Classified Model. PLS-DA is a regression model based on PLS, which integrates classified variables with spectral variables. In this study, 23 feature wavelengths were, respectively, selected using the CARS and SPA algorithms as spectral variables to establish a PLS-DA classified model. The number of optimal principal components of the SNV+MSC-FS-PLS-DA, SNV + MSC-CARS-PLS-DA, and SNV + MSC-SPA-PLSDA models were determined to be $14 \%, 13 \%$, and $14 \%$, respectively, based on the minimum RMSECV before modeling.

PLS-DA classified models based on different pretreatment methods of C-F, F- $\mathrm{T}_{\mathrm{o}}$, and $\mathrm{F}-\mathrm{T}_{\mathrm{t}}$ Tan mutton were established as shown in Table 2.

Table 2 shows that calibration and validation set accuracy of SNV + MSC-FS-PLS-DA based on the full spectrum removal of the noise band, which ranges from $1,010 \mathrm{~nm}$ to $1,678 \mathrm{~nm}$, can be up to $99 \%$ and $100 \%$, respectively, which shows that the full spectrum containing the 225 wavelength variables well preserves the effective information that was used in the model. The cross-validation error rate $(7 \%)$ of the model was lower than that of SNV + MSC-CARS-PLS-DA, which indicates the existence of invalid spectral variables among the 225 wavelengths. The variables selected using the CARS and SPA method only account for $10.2 \%$ of the full spectral variables, which indicates that these two methods can reduce the dimensions of the spectrum. The cross-validation error rate of SNV + MSC-CARS-PLS-DA was only 3\% (well below $7 \%$ ), and the calibration and validation set accuracy was $98 \%$ and $100 \%$, respectively. Its stability was better than SNV + MSC-FS-PLS-DA and SNV + MSC-SPA-PLS-DA as fewer optimal principal components were required for the former, which illustrates that the CARS algorithm can dispel redundant and irrelevant variables on the premise of maintaining better performance of the model. With fewer variables needed for modeling, the calculated speed of the SNV + MSC-CARS-PLS-DA model also increased. In contrast, the cross-validation error rate of SNV + MSC-SPA-PLSDA, respectively, decreased by $5 \%$ and $4 \%$, and the error rate of cross validation was higher than that of the other models.

Therefore, we concluded that CARS is a better method for extracting feature variables than SPA, with SNV + MSC-CARS-PLS-DA better than SNV + MSC-FSPLS-DA and SNV + MSC-SPA-PLS-DA. In addition, as 


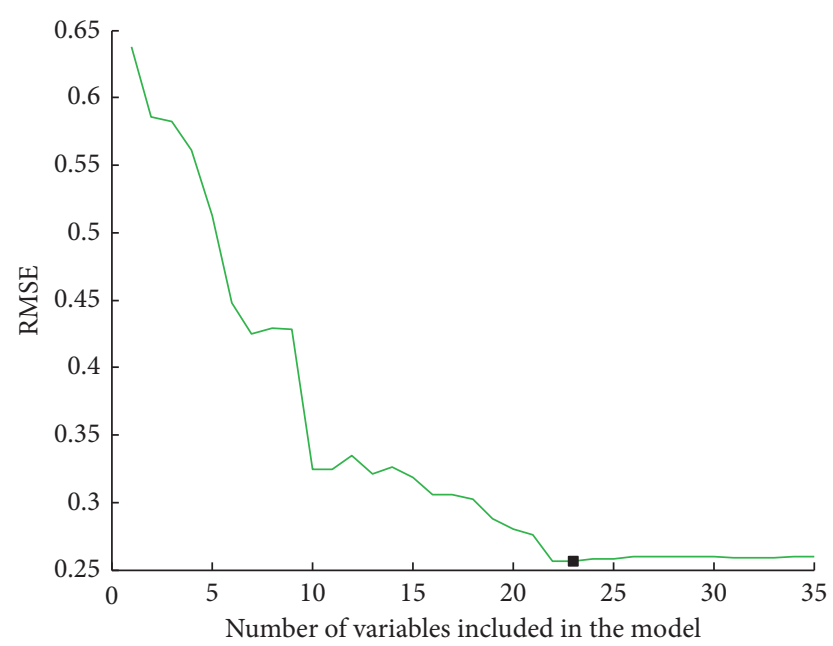

(a)

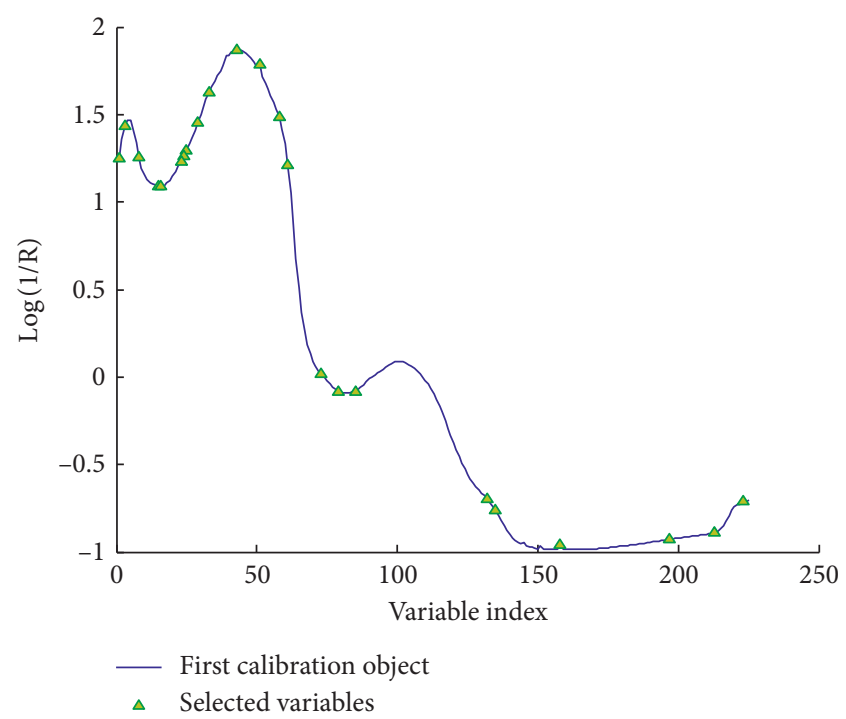

(b)

FIGURE 5: Feature wavelength extraction by SPA. (a) RMSE varies with the number of variables included in the model. (b) Feature wavelength extraction by SPA.

TABle 2: Classified models of C-F, F- $\mathrm{T}_{\mathrm{o}}$, and F- $\mathrm{T}_{\mathrm{t}}$ Tan mutton.

\begin{tabular}{lccccc}
\hline Modeling method & Wavelength number & Number of principal components & $\begin{array}{c}\text { Cross-validation } \\
\text { error rate }\end{array}$ & $\begin{array}{c}\text { Calibration } \\
\text { set accuracy }\end{array}$ & $\begin{array}{c}\text { Validation set } \\
\text { accuracy }\end{array}$ \\
\hline SNV + MSC-FS-PLS-DA & 225 & 14 & 0.07 & 0.99 & 1.00 \\
SNV + MSC-CARS-PLS-DA & 23 & 13 & 0.03 & 0.98 & 1.00 \\
SNV + MSC-SPA-PLS-DA & 23 & 14 & 0.09 & 0.94 & 0.96 \\
\hline
\end{tabular}

shown in Figure 6, C-F, F-T , and F-T Tan mutton can be well classified in the calibration and validation sets of SNV + MSC-CARS-PLS-DA.

\section{Conclusions}

Freezing is currently one of the most widely used methods to extend the shelf life and maintain the quality of Tan mutton. This study explicitly showed that the surface microstructure, texture (including hardness, gumminess, springiness, and chewiness) of C-F Tan mutton extensively deteriorated after F-T treatment, and further degradation occurred with increasing F-T time. The above changes during F-T storage results in a substantial decrease in the quality, taste, and commodity value of C-F Tan mutton.

To classify C-F and F-T Tan mutton and scientifically guide its storage and sale in the market, this study conducted a preliminary investigation on the internal and external changes in Tan mutton after F-T treatment. The hyperspectral data of C-F, F-T $\mathrm{T}_{\mathrm{o}}$, and $\mathrm{F}_{-} \mathrm{T}_{\mathrm{t}}$ Tan mutton were collected, pretreated by SNV, MSC, and SNV + MSC, and then feature spectral variables were extracted by CARS and SPA algorithms at 1,010 1,678 nm. SNV + MSC-CARS-PLS-DA based on 23 feature wavelengths was established and has superiorly classified performance (calibration set accuracy: 98\%) and the fastest computation speed.

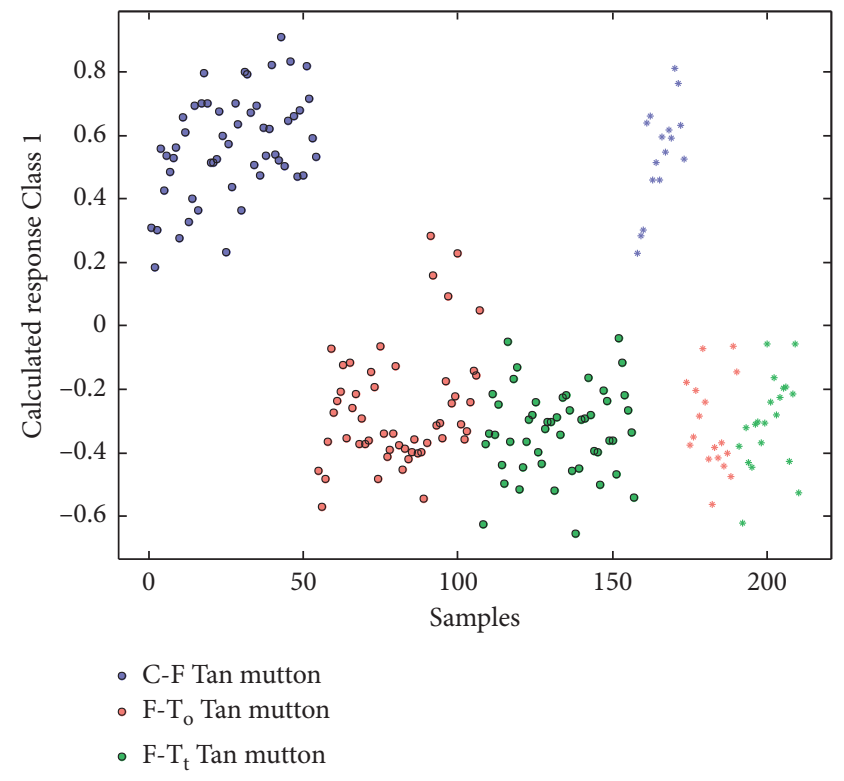

FIGURE 6: SNV + MSC-CARS-PLS-DA classification model.

There are also other changes in the F-T process such as fat degradation, protein denaturation, soluble nutrient loss, and surface chroma variations (including $\mathrm{L}^{*}, \mathrm{a}^{*}$, and $\mathrm{b}^{*}$ ). Additional comprehensive studies on the above changes in 
F-T mutton are warranted and, in combination with the results of this study, may accomplish quality classification of Tan mutton based on different F-T times.

\section{Data Availability}

The authors and affiliated agency allow disclosing the data to the public. The primary data used to support the findings of this study are available from the corresponding author or cofirst author upon request.

\section{Conflicts of Interest}

The authors declare that they have no conflicts of interest.

\section{Acknowledgments}

We thank LetPub (http://www.letpub.com) for its linguistic assistance during the preparation of this manuscript. This study was funded by the "Science, Engineering and Agricultural Projects of the Chinese Western First-Class University" (grant number ZKZD2017007).

\section{References}

[1] Z. S. Ma, "Analysis of volatile flavor substances in different parts of Ningxia Tan sheep," Graziery Veterinary Sciences, vol. 12, pp. 24-26, 2017.

[2] J. J. Song, "The choice of the development path of Yanchi county's first, second and third industry," China Southern Agricultural Machinery, vol. 16, pp. 229-230, 2018.

[3] J. Niu, G. S. Liu, S. Bo, R. M. Luo, and L. L. Wu, "Study on storage time of Tan mutton products in low temperature," China Condiment, vol. 7, pp. 33-49, 2017.

[4] O. Matteo, F. Luca, F. Pierantonio, and B. Massimiliano, "Foodstuff authentication from spectral data: toward a species-independent discrimination between fresh and frozen-thawed fish samples," Journal of Food Engineering, vol. 119 , no. 4, pp. 765-775, 2013.

[5] A. Pavlov, "Changes in the meat from aquaculture species during storage at low temperature and attempts for differentiation between thawed-frozen and fresh chilled meat," Bulgarian Journal of Veterinary Medicine, vol. 10, pp. 67-75, 2007.

[6] J. Carballo, S. Cofrades, M. T. Solas, and F. Jiménez-Colmenero, "High pressure/thermal treatment of meat batters prepared from freeze-thawed pork," Meat Science, vol. 54, no. 4, pp. 357-364, 2000.

[7] G. Duflos, B. Le Fur, V. Mulak, P. Becel, and P. Malle, "Comparison of methods of differentiating between fresh and frozen-thawed fish or fillets," Journal of the Science of Food and Agriculture, vol. 82, no. 12, pp. 1341-1345, 2002.

[8] P. Gottesmann and R. Hamm, "New biochemical methods of differentiating between fresh meat and thawed frozen meat," Fleischwirtschaft, vol. 63, pp. 219-220, 1983.

[9] J.-H. Park, C.-K. Hyun, S.-K. Jeong, M.-A. Yi, S.-T. Ji, and H.-K. Shin, "Use of the single cell gel electrophoresis assay (comet assay) as a technique for monitoring low-temperature treated and irradiated muscle tissues," International Journal of Food Science and Technology, vol. 35, no. 6, pp. 555-561, 2000.

[10] S. D. Evans, K. P. Nott, A. A. Kshirsagar, and L. D. Hall, "The effect of freezing and thawing on the magnetic resonance imaging parameters of water in beef, lamb and pork meat,"
International Journal of Food Science \& Technology, vol. 33, no. 3, pp. 317-328, 1998.

[11] G.-D. Kim, E.-Y. Jung, H.-J. Lim, H.-S. Yang, S.-T. Joo, and J.-Y. Jeong, "Influence of meat exudates on the quality characteristics of fresh and freeze-thawed pork," Meat Science, vol. 95, no. 2, pp. 323-329, 2013.

[12] X. Xia, B. Kong, Q. Liu, and J. Liu, "Physicochemical change and protein oxidation in porcine longissimus dorsi as influenced by different freeze-thaw cycles," Meat Science, vol. 83, no. 2, pp. 239-245, 2009.

[13] J. Ma, H. Pu, D.-W. Sun, W. Gao, J.-H. Qu, and K.-Y. Gao, "Application of Vis-NIR hyperspectral imaging in classification between fresh and frozen-thawed pork Longissimus Dorsi muscles," International Journal of Refrigeration, vol. 50, pp. 10-18, 2014.

[14] D. F. Barbin, D.-W. Sun, and C. Su, "NIR hyperspectral imaging as non-destructive evaluation tool for the recognition of fresh and frozen-thawed porcine longissimus dorsi muscles," Innovative Food Science \& Emerging Technologies, vol. 18, pp. 226-236, 2013.

[15] N. Barlocco, A. Vadell, F. Ballesteros, G. Galietta, and D. Cozzolino, "Predicting intramuscular fat, moisture and Warner-Bratzler shear force in pork muscle using near infrared reflectance spectroscopy," Animal Science, vol. 82, no. 1, pp. 111-116, 2006.

[16] D. Gerard and B. C. Dominique, "Discrimination between fresh and frozen-then-thawed beef $\mathrm{m}$. hgissimus dorsi by combined visible-near infrared reflectance spectroscopy," $A$ Feasibility Study Meat Science, vol. 35, pp. 353-363, 1997.

[17] W. J. Wang, S. L. Wang, X. G. He, and J. G. He, "Nondestructive detection of refrigerated time and moisture content in chilled mutton using hyperspectral imaging," Food Science, vol. 16, pp. 112-116, 2015.

[18] L. Jin, Study on the Internal Quality Detection Method of Ningxia Tan Mutton Based on Hyperspectral Technique, Ningxia University, Yinchuan, China, 2014.

[19] J. Y. Wang, Study on Non-Destructive Detection of Tan-Sheep Tenderness Based on Spectral Image Information Fusion, Ningxia University, Yinchuan, China, 2015.

[20] J. X. Ding, X. Y. Yang, M. M. Fang, and J. G. He, "Different kinds egg by Vis/NIR hyperspectral imaging technique," Chinese Journal of Luminescence (Electronic Version), vol. 3, pp. 395-397, 2018.

[21] M.-k. Minamiboso and S. Yokohama, "Comparison of connective tissue structure and muscle toughness of spotted mackerel Scomber australasicus and Pacific mackerel $S$. japonicus during chilled and frozen storage," Food Science and Technology, vol. 83, no. 1, pp. 133-199, 2017.

[22] S. Haga and T. Ohashi, "Heat-induced gelation of a mixture of myosin B and soybean protein," Agricultural and Biological Chemistry, vol. 48, no. 4, pp. 1001-1007, 1984.

[23] J. B. Li, Z. M. Guo, W. Q. Huang, B. H. Zhang, and C. J. Zhao, "Selection of variables and samples in NIR spectral prediction model of strawberry SSC content using CARS and SPA algorithms," Spectroscopy and Spectral Analysis, vol. 35, pp. $372-378,2015$.

[24] S. F. C. Soares, A. A. Gomes, M. C. U. Araujo, A. R. G. Filho, and R. K. H. Galvão, "The successive projections algorithm," TrAC Trends in Analytical Chemistry, vol. 42, pp. 84-98, 2013.

[25] J. A. Hobro, J. Kuligowski, M. Döll, and B. Lendl, "Differentiation of walnut wood species and steam treatment using ATR-FTIR and partial least squares discriminant analysis (PLS-DA)," Analytical and Bioanalytical Chemistry, vol. 398, no. 6, pp. 2713-2722, 2010. 
[26] W. S. Lin, C. M. Yang, and B. J. Kuo, "Classifying cultivars of rice (Oryza sativa L.) based on corrected canopy reflectance spectra data using the orthogonal projections to latent structures (O-PLS) method," Chemometrics and Intelligent Laboratory Systems, vol. 115, pp. 25-36, 2012.

[27] Y. Z. R. Chen, T. Z. Qi, and C. Zhang, "Study on construction strategy of correction set for total nitrogen spectral estimation model of paddy soil based on spectral transformation and kennard-stone algorithm," Spectroscopy and Spectral Analysis, vol. 35, pp. 2133-2139, 2017.

[28] Z. X. Xu, B. G. Li, and Q. Luo, "Progress in research of ice crystals in fast frozen food," Packaging and food machinery, vol. 36, pp. 63-66, 2018.

[29] Q. X. Zeng, Principle of Food Processing and Preservation, Chemical Industry Press, Beijing, China, 2015.

[30] J. Estrada-Solís, K. A. Figueroa-Rodríguez, B. Figueroa-Sandoval et al., "Microstructure and physical changes in the Mexican cooked lamb meat barbacoa made with chilled and frozen meat," Meat Science, vol. 118, pp. 122-128, 2016.

[31] J. P. Acker and L. E. Mcgann, "Cell-cell contact affects membrane integrity after intracellular freezing," Cryobiology, vol. 40, no. 1, pp. 54-63, 2000.

[32] C. H. Zhang, X. Li, and Y. Li, "Low-variable temperature and high humidity thawing improves lamb quality," Transactions of the Chinese Society of Agricultural Engineering, vol. 29, pp. 267-273, 2013.

[33] A. G. Xie, Spectral Characteristics of Pork Meat during the Freezing Process and Cold Storage and Rapid Detection of Product Quality, South China University of Technology, Guangzhou, China, 2015.

[34] F. Huang, Y. Li, J. Wu, J. Dong, and Y. Wang, "Identification of repeatedly frozen meat based on near-infrared spectroscopy combined with self-organizing competitive neural networks," International Journal of Food Properties, vol. 19, no. 5, pp. 1007-1015, 2015. 


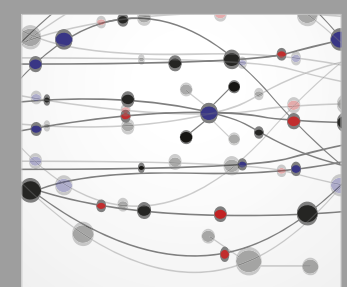

The Scientific World Journal
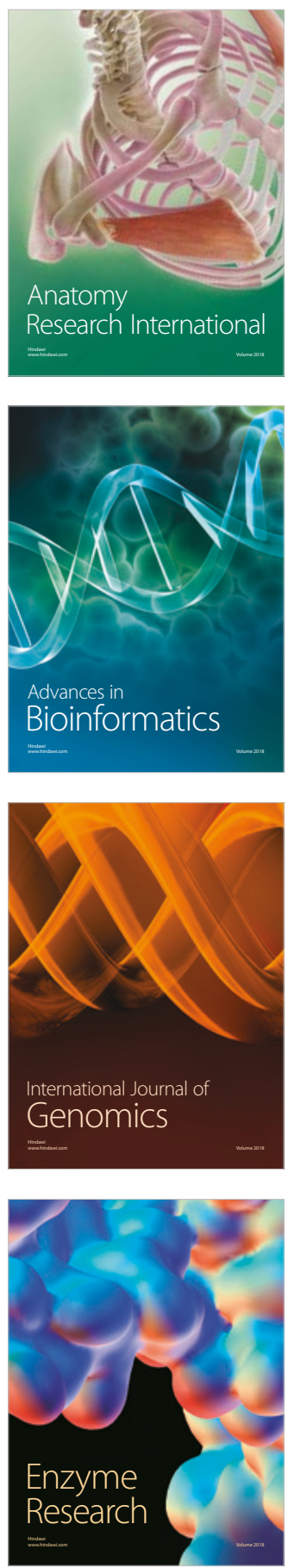
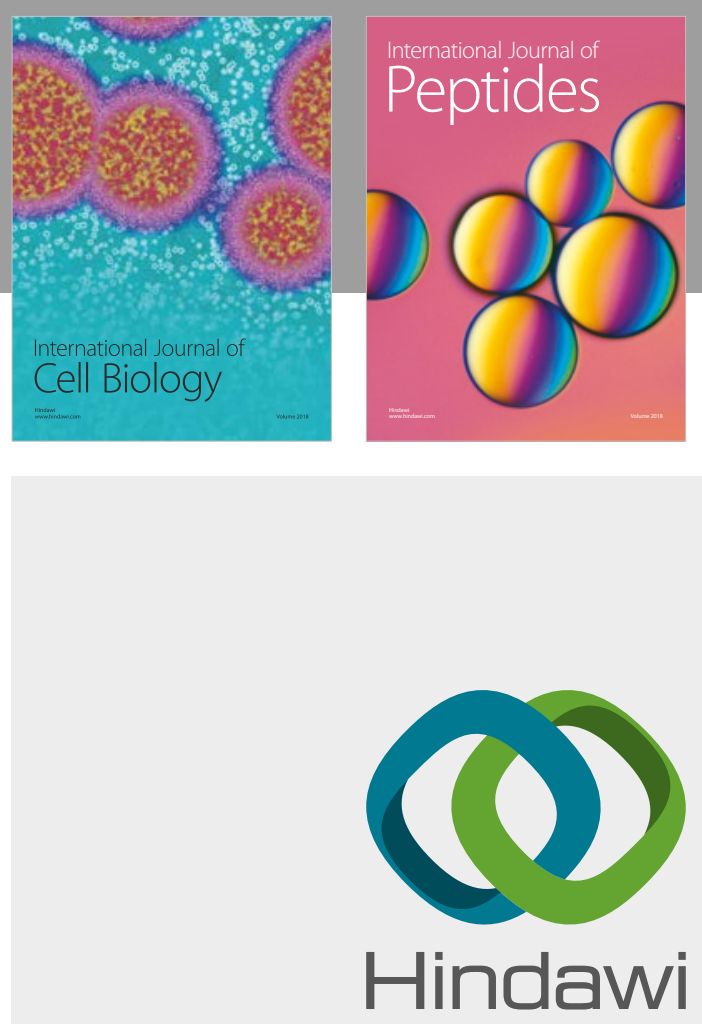

Submit your manuscripts at

www.hindawi.com
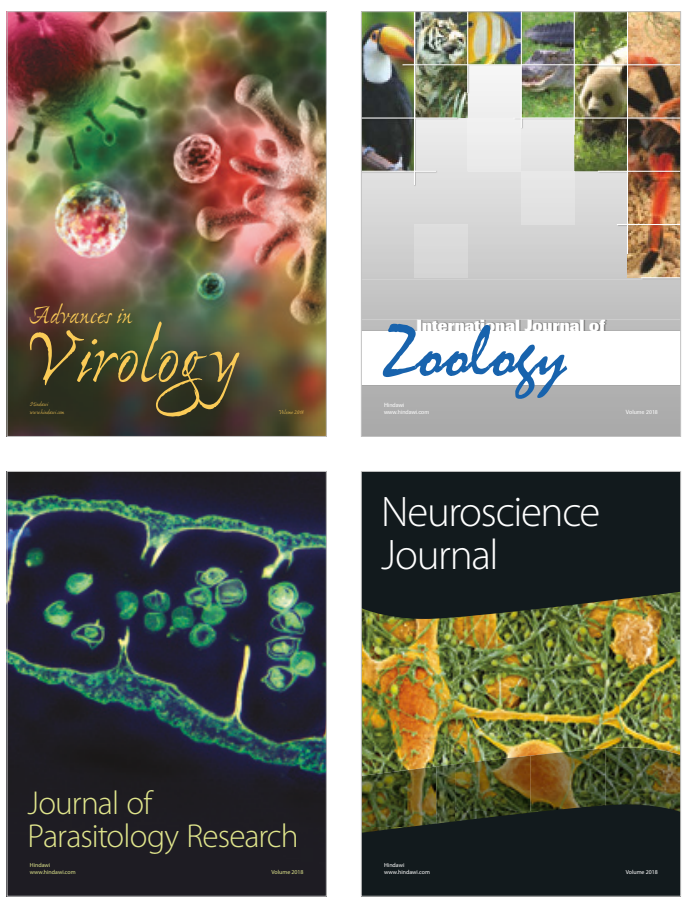
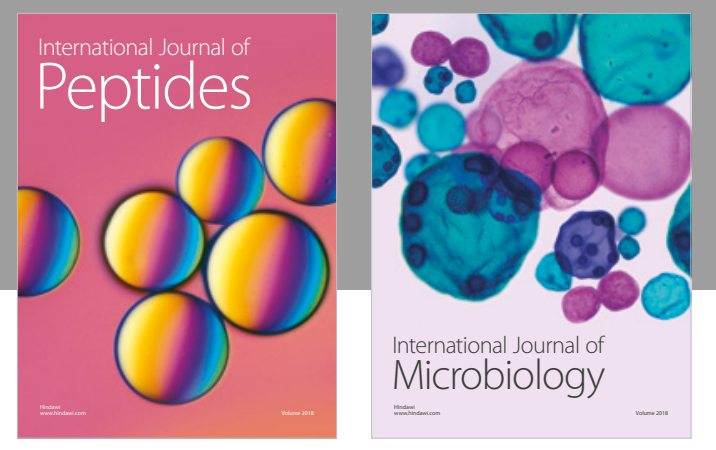

nternational Journal of Microbiology
Journal of
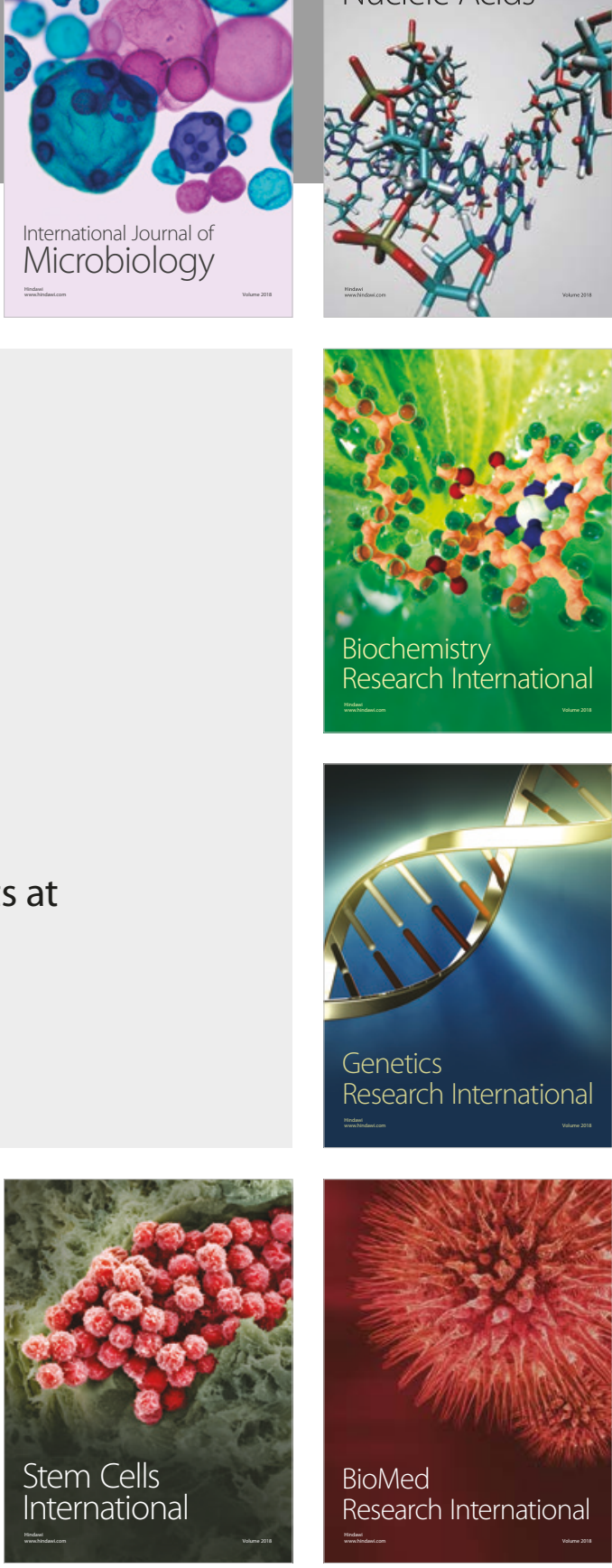
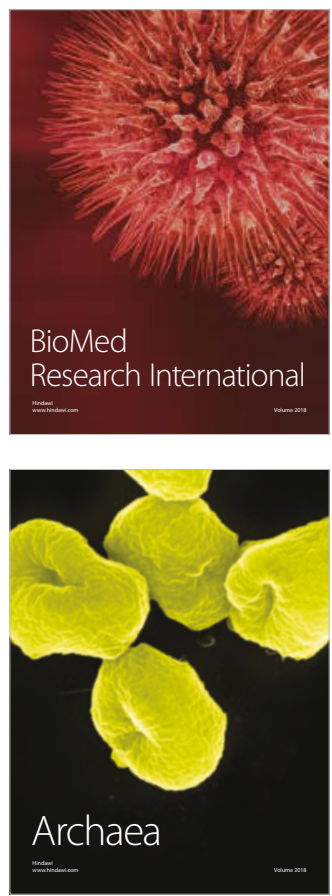